\title{
INFLUENCIA DE LA ANSIEDAD \\ Y LA FELICIDAD EN EL RENDIMIENTO ACADÉMICO INFANTIL (7-I2)
}

\section{Influence of anxiety and happiness over children's (7-I2) academic achievement}

Federico Pulido Acosta

Universidad de Granada

Correo-e: feanor_fede@hotmail.com

Francisco Herrera Clavero

Dpto. de Psicología Evolutiva y de la Educación. Universidad de Granada. Campus de Ceuta: Facultad de Educación, Economía y Tecnología. Universidad de Granada

Correo-e: fherrera@ugr.es

Recepción: 9 de febrero de 2018

Envío a informantes: 5 de marzo de 2018

Aceptación definitiva: 29 de abril de 2018

Resumen: Este trabajo tiene como objetivo analizar los niveles de ansiedad, felicidad y rendimiento académico (RA), en función de la edad, género, cultura y estrato socioeconómico, contemplando las relaciones que se pudieran dar entre estas variables. Se contó con 764 participantes, mayoritariamente de cultura-religión musulmana ( $74.2 \%)$. El $25.8 \%$ restante eran cristianos, siendo el $53.2 \%$ varones y el $46.8 \%$ mujeres. Las variables edad, género, cultura/religión y estatus influyen en Ansiedad y Felicidad. Se halló relación inversamente proporcional entre Ansiedad y Felicidad. En Rendimiento influyen edad, género, cultura/religión y estatus, encontrándose relación inversa entre Ansiedad y RA. Felicidad y RA son directamente proporcionales.

Palabras clave: estados emocionales; ansiedad; felicidad; Rendimiento Académico.

Aвstract: The main goal of this work is to analyze the types of anxiety, happiness and academic performance (AP), according to age, gender, culture and status, as well as the relationships between the variables. To make it possible we focused on 764 participants; $74.2 \%$ were Muslims and $25.8 \%$ Christians, $53.2 \%$ male and $46.8 \%$ fema- 
le. The results reflect that both variables are influenced by age, gender, culture and status. We found inversely proportional relationship between anxiety and happiness. AP is influenced by age, gender, culture and status. There is an inversely relationship between anxiety and AP. We have found a positive, significant association between happiness and AP.

KEY WORDs: emotional states; anxiety; happiness; academic performance.

\section{Introducción}

$\mathrm{N}$ O SE DEBEN OBVIAR LAS EMOCIONES y resulta fundamental en la vida saber reaccionar de manera adecuada ante ellas, ya que, considerándolas como un potencial, pueden transformarse en un arma muy importante con la que afrontar de manera exitosa la vida. En Pulido y Herrera (20I6) se relaciona el estado de ansiedad con emociones de carácter negativo.

Por este motivo, los objetivos del trabajo han sido analizar los tipos y niveles en ambos estados emocionales (ansiedad y felicidad), así como los de rendimiento académico, en función de la edad, género, cultura y estrato socioeconómico. También se pretende contemplar las relaciones que se pudieran dar entre las mencionadas variables, para una población infantil multicultural.

Consecuencias desfavorables pueden ir asociadas a este tipo de estados emocionales, pudiendo dificultar el adecuado desarrollo del autoconcepto, llegando a suponer una falta de aceptación por parte de los demás, dificultades en el proceso de socialización y malestar (Ortuño-Sierra, García-Velasco, Inchausti, Debbané y Fonseca-Pedrero, 2016). Los estados emocionales de carácter positivo, por su parte, implican el poner de relieve la importancia de potenciar la construcción de los aspectos positivos del ser humano, llenando la vida de las personas de felicidad y bienestar, un verdadero potencial para aplicarlo al alumnado (Barahona, Sánchez y Urchaga, 20I3). Así, se relacionan con mayores niveles de autoestima, lo que puede favorecer el desarrollo de conductas saludables y optimismo, permitiendo su crecimiento positivo (Moreno-Rosset, Arnal-Remón, Antequera-Jurado y Ramírez-Uclés, 2016).

Se puede comprobar un descenso progresivo en el diagnóstico de problemas de ansiedad a medida que el sujeto va aumentando la edad (Cazalla-Luna y Molero, 20I4; Gómez-Ortiz, Casas y Ortega-Ruiz, 20I6). De la misma forma, se puede intuir cierta independencia entre el nivel de felicidad manifestado por el sujeto y su edad (Carballeira, González y Marrero, 20I5). Otro de los elementos más relevantes dentro del campo de estudio de la ansiedad es su clara relación con el género, teniendo las mujeres niveles superiores de ansiedad (Moreno-Rosset et al., 20ı6; Pulido y Herrera, 20I6). Por su parte, algunos autores evidencian que son los varones los que son más proclives a manifestar niveles superiores de felicidad (Quiceno y Vinaccia, 2015). Resulta también relevante la pertenencia a un grupo cultural sobre los estados emocionales negativos, estando ligados a los valores de cada cultura, los cuales actúan como predictor de diferencias significativas en las manifestaciones emocionales (Caballero-Dominguez, Suárez-Colorado y Bruges-Carbono, 20I5; Giménez-Dasí y Quintanilla, 2009; y Pulido y Herrera, 2016). También existe influencia de la cultura sobre la manifestación de diferentes estados emocionales como la felicidad (Jia, Tian, He, Liu, Jin y Ding, 20I0; Quiceno y Vinaccia, 20I5). Todo lo que se ha comentado para 
la cultura, se relaciona de forma directa con el estatus socioeconómico, teniendo los estratos bajos mayores niveles de ansiedad (Pulido y Herrera, 20I6) y niveles menores de felicidad (Quiceno y Vinaccia, 20I5).

Por otro lado, aparece el rendimiento académico, que se refiere al nivel de conocimientos y destrezas escolares exhibidos por los estudiantes. En este caso, se van a emplear las calificaciones escolares como indicativo de este (Gómez-Castro, 1986).

Pulido y Herrera (2016) también establecen diferencias en función del género, siendo las mujeres las que presentan mejores resultados que los varones. La cultura es un factor mucho más determinante (Pulido y Herrera, 2or6), demostrándose diferencias en los niveles, en dos muestras de culturas diferentes. Otro de los elementos considerados como determinantes (Pulido y Herrera, 2016) es el estatus, que guarda una estrecha relación con el grupo cultural de pertenencia.

Se contempla también una relación entre ansiedad y rendimiento académico, dado que esta puede llegar a dificultar el desarrollo educativo, influyendo, de forma negativa en el mismo (Contreras, Espinosa, Esguerra, Haikal y Rodríguez, 2005), especialmente cuando aparece en niveles altos. Las relaciones también se describen entre la felicidad y este indicador del ámbito académico (Salanova, Martínez, Bresó, Llore y Grau, 2005), incidiendo en la importancia que tienen los trabajos en Psicología Positiva, centrada en el desarrollo óptimo y los aspectos positivos. Dentro de esta concepción, Perandones (20I5) destaca la evaluación subjetiva de la vida en términos de satisfacción y equilibrio. Los individuos que informan tener mayor felicidad presentarían mayor afecto positivo, menor afecto negativo y más satisfacción vital. Esto permite su crecimiento positivo, aminorando los estados emocionales negativos y mejorando el ajuste emocional y el éxito social y académico (Gutiérrez y Gonçalves, 20I3).

Considerando todo lo mencionado, se presenta el estudio llevado a cabo con una muestra de alumnos escolarizados en Centros de Educación Infantil y Primaria en la ciudad de Ceuta, donde conviven principalmente personas de cultura cristiana y musulmana. De esta forma, se pretende considerar qué variables sociodemográficas influyen en las variables presentadas y analizadas, para una población educativa multicultural.

\section{Método}

\section{I.I. Participantes}

Para llevar a cabo esta investigación se seleccionó una muestra integrada por 764 participantes, que se reparten entre siete centros en los que se imparte formación perteneciente a la Etapa Primaria y que reflejan las características del contexto pluricultural de Ceuta (en la Tabla i aparecen las características sociodemográficas, siendo representativa de la muestra educativa de Ceuta). El método para la selección de la muestra fue mediante un método aleatorio de sujetos disponibles. De esta manera, después de solicitar permiso por escrito, tanto a la Administración Educativa, como a la Dirección de los centros y, por supuesto, a los padres de cada niño, se procedió a la recogida de la información, contestando aquellos que lo desearon. Cada uno contestó, en primer lugar, a la información sobre las variables sociodemográficas y las calificaciones académicas, para pasar a contestar las cuestiones relativas a la ansiedad y, en 
último lugar, la felicidad. Finalmente, indicar que, en el presente trabajo, se cumplió con los estándares éticos.

TABLA i. Muestra en función de variables sociodemográficas

\begin{tabular}{|c|c|c|c|c|}
\hline \multirow{2}{*}{$\begin{array}{c}\text { Variables sociodemográficas } \\
\text { Edad }\end{array}$} & \multicolumn{4}{|c|}{ Media descriptiva } \\
\hline & $\mathrm{N}=764$ & $\begin{array}{c}\text { Media } \\
9.4 \mathrm{I}\end{array}$ & $\begin{array}{c}\text { D.T. } \\
\text { I.I9 }\end{array}$ & $\begin{array}{c}\text { Rango } \\
7-12\end{array}$ \\
\hline \multirow{3}{*}{ Cultura/Religión } & & & $\mathrm{N}$ & $\%$ \\
\hline & Cristianos & & 196 & 25.8 \\
\hline & Musulmanes & & 564 & 74.2 \\
\hline \multirow{2}{*}{ Género } & Varones & & 403 & 53.2 \\
\hline & Chicas & & 354 & 46.8 \\
\hline \multirow[t]{4}{*}{ Estatus } & Bajo & & 96 & 12.6 \\
\hline & Medio-Bajo & & 330 & 46.2 \\
\hline & Medio & & 245 & 32.1 \\
\hline & Alto & & 93 & $\mathrm{I} 2.2$ \\
\hline
\end{tabular}

\section{I.2. Instrumentos}

Para la evaluación de la ansiedad, se utilizó el Inventario de Ansiedad Estado-Rasgo para Niños (sTAic, Spielberger et al., 200I), aplicable a sujetos de entre 8 y i8 años.

Para la felicidad, se usó un instrumento elaborado para la ocasión, conformado por 40 ítems a los que se contesta con una escala tipo Lickert de 4 puntos (o -nada-, I - poco-, 2 -bastante- y 3 -mucho-).

Finalmente, se emplearon las calificaciones como medida del rendimiento académico, siguiendo el estudio de Gómez-Castro (1986).

\section{I.3. Análisis}

En primer lugar, se comenzó por los cálculos de representatividad muestral en relación a la población escolarizada. Se continuó con el análisis de las propiedades psicométricas de los cuestionarios empleados, para pasar al análisis inferencial, comenzando por el análisis regresional (regresiones múltiples paso a paso o «stepwise»). Como variables criterio se utilizaron cada una de las variables de estudio (Ansiedad, Felicidad y Rendimiento Académico). Dentro de estas, para hacer el análisis más específico y detallado se consideraron también cada una de las áreas académicas (especificadas en el párrafo anterior) y los factores que integran ambos estados emocionales, obtenidos a través del AFE. En el análisis regresional de cada una de ellas, se emplearon las variables sociodemográficas mencionadas como predictoras (independientes). Además de las variables sociodemográficas se incluyeron (como independientes) aquellas variables de estudio que no se consideraban como variable criterio (dependiente) y las áreas académicas (rendimiento) o factores (ansiedad y felicidad) que la conformaban. Para determinar la función de regresión se consideró el último paso y, dentro de él, en el orden de entrada de las variables en la función de regresión, el valor de cada variable, su 
significación, el índice de regresión $(R)$, el coeficiente de determinación múltiple $\left(R^{2}\right)$ y la varianza explicada de la variable más importante (la que más porcentaje - $\beta$ - explica). A continuación se procedió al análisis inferencial, empleando la prueba $t$ de Student de diferencias de medias para muestras independientes para aquellas variables dicotómicas (género y cultura), mientras que para el resto (curso-edad, estatus, ansiedad y felicidad) se empleó el análisis de la varianza (ANOva de un factor). Al mismo tiempo, se utilizó la prueba de Chi-cuadrado de Pearson, aprovechando los resultados obtenidos dentro de las tablas de contingencia que se hicieron para la estadística descriptiva.

\section{Resultados}

Se comienza con la fiabilidad para la medida de la ansiedad. Se evaluó a través de la prueba de Cronbach. Así, la consistencia interna del cuestionario fue de .882, incluyendo todos los ítems. En el apartado relacionado con la varianza factorial se emplearon diferentes Análisis Factoriales Exploratorios (AFE). En este sentido, los factores obtenidos fueron 6 (sus características aparecen en la Tabla 2). El primero se relaciona con los Rasgos emocionales negativos. El segundo de los factores se relaciona con diferentes Preocupaciones y dificultades. El tercer factor se vinculó con los Estados emocionales positivos. El cuarto factor pertenece a la categoría de Sensaciones de ansiedad. El quinto factor es Estados emocionales negativos y el último de los seis factores se relaciona con Estados emocionales contrapuestos. Entre todas las categorías se consigue una varianza total explicada del $51.27 \%$.

TABLA 2. Fiabilidad y varianza e ítems de la agrupación por factores del staIC

\begin{tabular}{|c|c|c|c|c|}
\hline \multicolumn{5}{|c|}{ CONSISTENCIA INTERNA DEL CUESTIONARIO } \\
\hline & STAIC & $\alpha$ de Cronbach & & .882 \\
\hline \multicolumn{5}{|c|}{ VARIANZA DE FACTORES DE CUESTIONARIO STAIC } \\
\hline & FACTOR & N.o ítems & V. explicada & Suma total \\
\hline \multirow{6}{*}{$\begin{array}{l}\text { 是 } \\
\text { 島 } \\
\text { 㫘 }\end{array}$} & Emociones negativas & 9 & $21.58 \%$ & \multirow{6}{*}{$\begin{array}{c}40 \text { ítems } \\
5 \mathrm{I} .27 \% \text { en la varianza } \\
\text { total explicada }\end{array}$} \\
\hline & Preocupaciones y dificultades & 9 & $10.60 \%$ & \\
\hline & Estados emocionales positivos & 9 & $9.119 \%$ & \\
\hline & Sensaciones de ansiedad & 5 & $5.774 \%$ & \\
\hline & Estados emocionales negativos & 5 & $3.246 \%$ & \\
\hline & Estados emocionales contrapuestos & 3 & $2.968 \%$ & \\
\hline
\end{tabular}

Para la felicidad, los resultados que hacen referencia a la consistencia interna del instrumento dan una fiabilidad, establecida por un de Cronbach de .880. En el apartado relacionado con la varianza factorial (AFE), los factores obtenidos fueron 6 (sus características aparecen en la Tabla 3). El primero se relaciona con las Condiciones en la vida que son de carácter negativo. El segundo de los factores se relaciona con la Satisfacción ante la vida. El tercer factor se relaciona con las Condiciones positivas en la vida. El cuarto factor se relaciona con la intención del sujeto de producir Cambios en su vida, mientras que el quinto queda vinculado con la intención del sujeto de No introducir Cambios en su vida. El último factor se relaciona con Preocupaciones en la vida. Entre todos los factores suman una varianza total explicada del $54.591 \%$. 
TABLA 3. Fiabilidad y varianza e ítems de la agrupación por factores del instrumento para la evaluación de la felicidad

\begin{tabular}{|c|c|c|c|c|}
\hline \multicolumn{5}{|c|}{ CONSISTENCIA INTERNA DEL CUESTIONARIO } \\
\hline & FELICIDAD & $\alpha$ de Cronbach & & \\
\hline \multicolumn{5}{|c|}{ VARIANZA DE FACTORES DE CUESTIONARIO FELICIDAD } \\
\hline & FACTOR & N.o ítems & V. explicada & Suma total \\
\hline \multirow{6}{*}{ 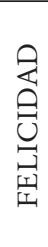 } & Condiciones de vida negativas & 12 & $24.469 \%$ & \multirow{6}{*}{$\begin{array}{c}40 \text { ítems } \\
54.591 \% \text { en la } \\
\text { varianza total } \\
\text { explicada }\end{array}$} \\
\hline & Satisfacción con la vida & IO & $12.14 \%$ & \\
\hline & Condiciones de vida positivas & 6 & $6.252 \%$ & \\
\hline & Cambios en la vida & 4 & $5.828 \%$ & \\
\hline & No cambios en la vida & 5 & $3.019 \%$ & \\
\hline & Preocupaciones en la vida & 3 & $2.871 \%$ & \\
\hline
\end{tabular}

\section{I. Estados emocionales}

Los alumnos de primaria, en relación a la variable Ansiedad, demuestran puntuaciones bajas y muy bajas. Por contra, en relación a la variable Felicidad, se puede observar que se mueve en puntuaciones altas y muy altas.

Se inicia la descripción de los resultados de la muestra de alumnos, en función de la variable Curso/Edad. El grupo en el que se registran los mayores niveles de ansiedad es en el de los alumnos de $3 .^{\circ}$ de Primaria (8-9 años con una media de 40.03). El siguiente curso, por nivel, es $4 .^{\circ}$ (9-Io años, reflejaron 33.70 de media), seguido de $2 .^{\circ}$ (7-8 años, 32.46). Después aparecería $5 .^{\circ}$ de Primaria (IO-II años, con una media de 30.54), seguido por el alumnado de $6 .^{\circ}$ (II-I2 años y 28.20 de media). Por contra, en Felicidad, $5 .^{\circ}$ de Primaria (IO-II) es el nivel educativo en el que se reflejan los mejores resultados de felicidad (88.47). Le sigue el 6. (II-I2 años, con una media cercana de 87.45). Con puntuaciones más bajas aparece $4 .^{\circ}$ (9-Io años y una media de 83.39). Los alumnos con peores resultados en felicidad son los de $3 .^{\circ}$ (77.37 y entre 8 y 9 años) y, sobre todo, $2 .^{\circ}$ (7-8 años) con una media de 76.46. Considerando el Análisis de la Varianza (Anova de un factor, en la Tabla 4), en Ansiedad Total y en sus factores Rasgos emocionales negativos, Preocupaciones y dificultades, Estados emocionales positivos, Sensaciones de Ansiedad, Estados emocionales negativos y Estados emocionales contrapuestos, se encontraron diferencias estadísticamente significativas (las pruebas post hoc determinan diferencias entre todos los cursos, excepto con el $2 .^{\circ}$ ). Lo mismo ocurrió con la Felicidad Total y todas sus categorías (Condiciones de vida negativas, Satisfacción con la vida, Condiciones de vida positivas, Cambios en la vida, No cambios en la vida y Preocupaciones en la vida). Para esta variable, las diferencias se dan entre $2 .^{\circ}$ y el resto de cursos, excepto $3 .^{\circ}(8-9)$, entre $3 .^{\circ}$ y el resto y entre $4 .^{\circ}$ y $5 .^{\circ}$ y $6 .^{\circ}$ (como indican las pruebas post hoc).

Considerando la variable género, se puede ver que los niños tienen un nivel más alto que las niñas en cuanto a las puntuaciones en Ansiedad, tal y como reflejan las medias de ambos ( 34.42 los varones y 31.88 las mujeres). Esta realidad se repite también en cada uno de los factores que integran esta variable. Sin embargo, en Felicidad los chicos presentan puntuaciones más bajas (82.5) que las chicas (85.53). Lo mismo ocurre en todos los factores. Una vez hecha esta primera aproximación, se empleó la prueba 
INFLUENCIA DE LA ANSIEDAD Y LA FELICIDAD EN EL RENDIMIENTO ACADÉMICO INFANTIL (7-I 2)

FEDERICO PULIDO ACOSTA Y FRANCISCO HERRERA CLAVERO

TABla 4. ANOva Estados emocionales por Edad

\begin{tabular}{|c|c|c|c|c|c|c|c|}
\hline Variable & $\mathrm{N}$ & Perdidos & & Media & Des. Tip. & $\mathrm{F}$ & $\mathrm{P}$ \\
\hline \multicolumn{8}{|c|}{ Ansiedad } \\
\hline \multirow{5}{*}{ Ansiedad Total } & I5 & o & $2 .^{\circ}(7-8)$ & 32.4667 & 8.32266 & \multirow{5}{*}{15.676} & \multirow{5}{*}{.000} \\
\hline & $2 \mathrm{II}$ & 2 & $3 .^{\circ}(8-9)$ & 40.0379 & 17.02515 & & \\
\hline & 168 & o & $4 \cdot^{\circ}($ IO-II) & 33.7024 & I5.9357I & & \\
\hline & I78 & o & $5 .^{\circ}(\mathrm{IO}-\mathrm{II})$ & 30.5449 & 15.97246 & & \\
\hline & 185 & 3 & 6. ${ }^{\circ}(\mathrm{II}-\mathrm{I} 2)$ & 28.2054 & $\mathrm{I} 4.68662$ & & \\
\hline \multicolumn{8}{|c|}{ Felicidad } \\
\hline \multirow{5}{*}{ Felicidad Total } & I5 & o & $2 .^{\circ}(7-8)$ & 76.4667 & 16.54374 & \multirow{5}{*}{16.411} & \multirow{5}{*}{.000} \\
\hline & $2 \mathrm{II}$ & 2 & $3 .^{\circ}(8-9)$ & 77.3744 & 16.91230 & & \\
\hline & 168 & o & $4 \cdot^{\circ}(\mathrm{IO}-\mathrm{II})$ & 83.3952 & 15.71545 & & \\
\hline & I78 & o & $5 .^{\circ}$ (IO-II) & 88.4719 & $\mathrm{I} 3.468 \mathrm{I} 8$ & & \\
\hline & 185 & 3 & $6^{\circ}{ }^{\circ}(\mathrm{II}-\mathrm{I} 2)$ & 87.4590 & $15.5813 \mathrm{I}$ & & \\
\hline
\end{tabular}

$t$ de Student (Tabla 5). Considerando este análisis, las diferencias vuelven a ser estadísticamente significativas en Ansiedad Total y en los factores Estados emocionales positivos, Sensaciones de Ansiedad y Estados emocionales negativos. En el caso de la Felicidad, aparecen en las puntuaciones Totales, Condiciones de vida positivas y Cambios en la vida.

Tabla 5. Prueba t de Student Estados emocionales por Género

\begin{tabular}{|c|c|c|c|c|c|c|}
\hline Variable & $\mathrm{N}$ & Perdidos & Media & Des. Tip. & $\mathrm{t}$ & $\mathrm{P}$ \\
\hline \multicolumn{7}{|c|}{ Ansiedad } \\
\hline \multirow{2}{*}{ Ansiedad Total } & $4 \mathrm{OI}$ & $2 \quad$ Varones & 34.4214 & 16.06999 & \multirow{2}{*}{4.522} & \multirow{2}{*}{.034} \\
\hline & 352 & $2 \quad$ Mujeres & 31.8835 & I6.64239 & & \\
\hline \multicolumn{7}{|c|}{ Felicidad } \\
\hline \multirow{2}{*}{ Felicidad Total } & 399 & $4 \quad$ Varones & 82.5088 & 16.21415 & \multirow{2}{*}{6.637} & \multirow{2}{*}{.oro } \\
\hline & $35 \mathrm{I}$ & $3 \quad$ Mujeres & 85.5356 & 15.87229 & & \\
\hline
\end{tabular}

Teniendo en cuenta la cultura/religión, el alumnado perteneciente a la cultura/religión musulmana manifiesta niveles más altos de ansiedad (la media de los cristianos es 31.16 mientras que los musulmanes alcanzan 34.02). Esta situación también se refleja en los factores Rasgos emocionales negativos, Estados emocionales positivos, Sensaciones de Ansiedad y Estados emocionales negativos. La cultura también resulta fundamental para la variable Felicidad, apareciendo diferencias entre ambas, donde los cristianos (87.5) presentan una media mayor a los musulmanes (82.58), lo que indica un nivel de felicidad superior. Esta realidad se repite en diferentes factores. Considerando la prueba $t$ de Student (Tabla 6), se pueden confirmar diferencias estadísticamente significativas en la variable Ansiedad Total, Rasgos emocionales negativos, Estados emocionales positivos, Sensaciones de Ansiedad y Estados emocionales negativos, así como en Felicidad Total y en las categorías Condiciones de vida negativas, Satisfacción con la vida y Preocupaciones en la vida. 
TABla 6. Prueba t Estados emocionales por Cultura

\begin{tabular}{|c|c|c|c|c|c|c|}
\hline Variable & $\mathrm{N}$ & Perdidos & Media & Des. Tip. & $\mathrm{t}$ & $\mathrm{P}$ \\
\hline \multicolumn{7}{|c|}{ Ansiedad } \\
\hline \multirow{2}{*}{ Ansiedad Total } & 196 & $\circ \quad$ Cristiana & 31.1633 & 16.99212 & \multirow{2}{*}{$4 \cdot 447$} & \multirow{2}{*}{.035} \\
\hline & 560 & 4 Musulmana & 34.0232 & I6.10768 & & \\
\hline \multicolumn{7}{|c|}{ Felicidad } \\
\hline \multirow{2}{*}{ Felicidad Total } & 196 & o Cristiana & 87.5000 & 15.22127 & \multirow{2}{*}{13.688} & \multirow{2}{*}{.000} \\
\hline & 557 & 7 Musulmana & 82.5835 & I6.26519 & & \\
\hline
\end{tabular}

Si se considera el estatus socioeconómico, en Ansiedad, se observa una distribución desorganizada (consideradas las pruebas post hoc las diferencias se dan entre el estatus medio - puntuaciones más bajas- y el medio-bajo -puntuaciones más altas- y el alto) en Ansiedad Total $(p=.042)$ : por media, los niveles más bajos aparecen en el estatus medio (con una media de 30.9I), seguido por el alto (34.27). Después aparecería el estatus bajo, alcanzando una media de 34.62. Solo es estatus medio-bajo (34.64) presenta niveles ligeramente superiores de ansiedad. Con respecto a la variable Felicidad (las diferencias estadísticamente significativas se dan entre medio y el bajo y el medio-bajo, según las pruebas post hoc), se observa una distribución más organizada, ya que, por media, los niveles más altos aparecen en los estatus altos (medio con una media de 86.8I y alto con 83.95). El nivel medio-bajo (82.96) y el bajo (78.75) alcanzan resultados inferiores. En Ansiedad Total, Estados emocionales positivos, Sensaciones de Ansiedad, Estados emocionales negativos, Estados emocionales contrapuestos, Felicidad, Condiciones de vida negativas, Satisfacción con la vida, Condiciones de vida positivas y Cambios en la vida las diferencias fueron estadísticamente significativas (en la Tabla 7 aparecen los resultados del ANOva para las puntuaciones totales de ambas variables).

TABLa 7. ANOva Estados emocionales por Estatus

\begin{tabular}{|c|c|c|c|c|c|c|}
\hline Variable & $\mathrm{N}$ & Perdidos & Media & Des. Tip. & $\mathrm{F}$ & $\mathrm{P}$ \\
\hline \multicolumn{7}{|c|}{ Ansiedad } \\
\hline \multirow{4}{*}{ Ansiedad Total } & 96 & Bajo & 34.6250 & 17.88516 & \multirow{4}{*}{2.748} & \multirow{4}{*}{.042} \\
\hline & 328 & Medio-Bajo & 34.6463 & I6.0I25I & & \\
\hline & 243 & Medio & 30.9177 & I5. 77428 & & \\
\hline & 92 & Alto & 34.2717 & 17.83323 & & \\
\hline \multicolumn{7}{|c|}{ Felicidad } \\
\hline \multirow{4}{*}{ Felicidad Total } & 96 & Bajo & 78.7500 & $16.96 \mathrm{1} 26$ & \multirow{4}{*}{6.333} & \multirow{4}{*}{.000} \\
\hline & 328 & Medio-Bajo & 82.9665 & I5.86397 & & \\
\hline & 243 & Medio & 86.8167 & 15.31509 & & \\
\hline & 92 & Alto & 83.9565 & I7.I4894 & & \\
\hline
\end{tabular}

Considerando el cálculo regresional, la variable Felicidad Total se encuentra relacionada con la Ansiedad Total, así como dos factores pertenecientes a esta variable: Estados emocionales negativos y Preocupaciones y dificultades. La primera actúa de forma negativa, lo que indica una relación inversamente proporcional, a pesar de que 
ambos factores lo hacen de forma positiva. También se encuentra relacionada con la media del Rendimiento Académico y la Edad. Todos estos últimos toman valores positivos. Entre todas las predictoras explican el $40.5 \%$ de la varianza total $\left(R^{2}=.405\right)$. Una mayor fuerza es ejercida por la Ansiedad Total, que alcanza un coeficiente de regresión estandarizado de $74 . \mathrm{I} \%(\beta=.74 \mathrm{I})$. La función descrita sería la siguiente, lo que se une a los coeficientes que aparecen a continuación:

Felicidad Total $=60.6 \mathrm{I} 3-.72 \mathrm{I}($ Ansiedad Total $)+\mathrm{I} .875($ Media Rendimiento $)+.599$ (Preocupaciones) + .9II (Estados negativos) + I.3I4 (Edad)

Considerando los niveles de Felicidad, en función de la Ansiedad total mostrada por el sujeto, se puede observar una relación inversamente proporcional. Así, los sujeto que manifiestan un mayor nivel de felicidad son aquellos que presentan muy poca ansiedad (cuya media es de 91.53). A estos les seguiría los que presentan poca ansiedad (78.09) y, posteriormente, los que presentan bastante (63.34). Finalmente, con los niveles más bajos (63.00) aparece el único individuo que manifiesta mucha ansiedad. Para confirmar la relación inversamente proporcional entre ambas variables, se realizó un ANOva de un factor (Tabla 9). Se encontraron diferencias estadísticamente significativas (el análisis post hoc indicó diferencias entre todos los grupos) en Felicidad Total, Condiciones de vida negativas, Satisfacción con la vida, Condiciones de vida positivas, Cambios en la vida, No cambios en la vida y Preocupaciones en la vida.

Tabla 8. Anova Felicidad por Ansiedad Total

\begin{tabular}{|c|c|c|c|c|c|c|c|}
\hline Variable & $\mathrm{N}$ & Perdidos & & Media & Des. Tip. & F & $\mathrm{P}$ \\
\hline \multicolumn{8}{|c|}{ Ansiedad Total } \\
\hline \multirow{4}{*}{ Felicidad Total } & $38 \mathrm{I}$ & $\mathrm{Mu}$ & luy Poca Ansiedad & 91.5354 & II. $4805 \mathrm{I}$ & \multirow{4}{*}{100.929} & \multirow{4}{*}{.000} \\
\hline & 319 & Poc & ca Ansiedad & 78.0909 & I5.42688 & & \\
\hline & 55 & Bas & astante Ansiedad & 63.3455 & I6.61238 & & \\
\hline & I & $\mathrm{Mu}$ & Iucha Ansiedad & 63.0000 & & & \\
\hline
\end{tabular}

\subsection{Rendimiento Académico}

En el caso del Rendimiento, el resultado más habitual (31.8\%) es el notable. La siguiente calificación, por porcentaje, es el «suficiente» (23.1\%). A continuación aparecen las calificaciones de «bien» (con un I8.5\%) y sobresaliente (I5.2\%). La calificación con porcentajes más bajos (II.5\%) es el suspenso.

Considerando la variable Rendimiento, se inicia la descripción en función de la variable Curso/Edad. El curso con la media más alta es $5 .{ }^{\circ}(6.88)$. El siguiente sería $3 .^{\circ}$ (6.85) y después $6 .^{\circ}$, que alcanza los 6.76 puntos de media. Los resultados más bajos se dan en $4 .^{\circ}$ de Primaria (6.38). El anova (Tabla io) muestra diferencias estadísticamente significativas en las materias de Ciencias Sociales, Naturales y Religión. En el resto de materias, así como en la media las diferencias no fueron estadísticamente significativas.

Considerando el género, se puede comprobar que las chicas presentan niveles superiores, en cuanto a las calificaciones, alcanzando una media de 7.044, mientras que 
los chicos llegan hasta 6.49. De esta manera, se puede hablar de resultados superiores en las chicas, algo que se confirma en el resto de materias. En este caso, la prueba t de Student muestra diferencias estadísticamente significativas en la Media general, Lengua Castellana, Matemáticas, Sociales, Inglés, Naturales y Religión, teniendo mejor rendimiento las chicas.

Si se tiene en cuenta la Media de Rendimiento, en función de la cultura/religión, se puede decir que las calificaciones son claramente superiores entre los cristianos, ya que superan a los musulmanes en Rendimiento medio general. La media de los cristianos es de 7.68, mientras que la de los musulmanes es de 6.33. Esta realidad se ve reforzada en cada una de las materias, confirmándose por la prueba t de Student (Tabla Io), dado que las diferencias fueron estadísticamente significativas en la Media de Rendimiento y en todas las materias: Lengua Castellana, Matemáticas, Sociales, Inglés, Naturales y Religión.

Si se considera el estatus, se observa que las calificaciones aumentan progresivamente a medida que se asciende en el mismo. De esta manera, en el estatus alto es en el que aparecen niveles superiores, seguido del medio. Estos presentan una media de 7.46 y 7.II respectivamente. Las calificaciones descienden bastante para los otros dos grupos. El medio-bajo (6.36) y el bajo (6.I7) presentan las calificaciones más bajas. Una vez hecha esta primera aproximación, el Análisis de la Varianza (ANOva de un factor en la Tabla Io) demostró diferencias estadísticamente significativas en la Media general, así como en las áreas específicas: Lengua Castellana, Matemáticas, Sociales, Inglés, Naturales y Religión. Las diferencias, según el análisis post hoc, se dan entre el estatus bajo y el resto (excepto el medio-bajo) y entre el alto y el resto (excepto el medio).

TABLA 9. ANOva/prueba t Rendimiento por variables sociodemográficas

\begin{tabular}{|c|c|c|c|c|c|c|c|}
\hline Variable & $\mathrm{N}$ & Perc & didos & Media & Des. Tip. & $\mathrm{F} / \mathrm{t}$ & $\mathrm{P}$ \\
\hline \multicolumn{8}{|c|}{ Curso } \\
\hline \multirow{4}{*}{$\begin{array}{c}\text { Media } \\
\text { Rendimiento } \\
\text { Académico }\end{array}$} & II2 & IOI & $3 .^{\circ}(8-9)$ & 6.8595 & 2.02065 & \multirow{4}{*}{ I.876 } & \multirow{4}{*}{.133} \\
\hline & I6I & 7 & $4 .^{\circ}(9-10)$ & 6.3836 & $2.06 \mathrm{II} 7$ & & \\
\hline & I70 & 8 & $5 .^{\circ}$ (IO-II) & $6.868 \mathrm{I}$ & I. 68822 & & \\
\hline & I8I & 7 & $6 .^{\circ}(\mathrm{II}-\mathrm{I} 2)$ & 6.7703 & I. 55335 & & \\
\hline \multicolumn{8}{|c|}{ Género } \\
\hline \multirow{2}{*}{$\begin{array}{c}\text { Media } \\
\text { Rendimiento }\end{array}$} & 335 & 68 & Varones & 6.4946 & I. 88837 & \multirow{2}{*}{12.129} & \multirow{2}{*}{.001} \\
\hline & 294 & 60 & Mujeres & 7.0449 & I. 74058 & & \\
\hline \multicolumn{8}{|c|}{ Cultura } \\
\hline \multirow{2}{*}{$\begin{array}{c}\text { Media } \\
\text { Rendimiento }\end{array}$} & 173 & 23 & Cristiana & 7.6872 & I. 58655 & \multirow{2}{*}{68.864} & \multirow{2}{*}{.000} \\
\hline & 457 & 107 & Musulmana & 6.3319 & I. 79476 & & \\
\hline \multicolumn{8}{|c|}{ Estatus } \\
\hline \multirow{4}{*}{$\begin{array}{c}\text { Media } \\
\text { Rendimiento }\end{array}$} & 67 & 29 & Bajo & 6.1760 & I. 57436 & \multirow{4}{*}{ I3.447 } & \multirow{4}{*}{.000} \\
\hline & 283 & 47 & Medio-Bajo & 6.3665 & I. 79386 & & \\
\hline & 190 & 55 & Medio & 7.II42 & I.91486 & & \\
\hline & 92 & $\mathrm{I}$ & Alto & 7.4659 & I. 71674 & & \\
\hline
\end{tabular}

El Rendimiento Académico está bajo la influencia de varias variables. La que ejerce un poder más determinante es la Edad, cuyo coeficiente de regresión estandarizado 
es del 29.4\% $(\beta=.294)$. Esta variable actúa de manera inversamente proporcional. Por otra parte, también ejercen influencia las variables Cultura/Religión y Felicidad Total y el factor Cambios en la vida. Solamente una (Felicidad) actúa de manera positiva (directamente proporcional). La Cultura/Religión (esto indica una calificación más baja en los musulmanes, dado que se asoció esta cultura con el valor 2, mientras que los cristianos fueron asignados al valor I), la Edad y el factor actúan de manera negativa. El resto de variables quedan excluidas, explicando entre todas las predictoras el $42.5 \%$ de la varianza total $\left(R^{2}=.425\right)$. La influencia de estas variables es intensa, tal y como indica el coeficiente de correlación $(R=.652)$.

Media Rendimiento $=6.7 \mathrm{I} 2-.83 \mathrm{I}($ Cultura/Religión $)-.48 \mathrm{I}($ Edad $)+.014($ Felicidad Total) - 0.54 (Cambios vida)

Considerando el Análisis de la Varianza (ANova de un factor, en la Tabla I2), con la variable Felicidad (Total) como factor, se encontraron diferencias estadísticamente significativas en la Media general, así como en las áreas Lengua Castellana, Matemáticas, Ciencias Sociales, Inglés, Naturales y Religión. El análisis post hoc indica una relación directamente proporcional entre los niveles de felicidad y las calificaciones académicas (ya que se dan entre los que reflejan mucha felicidad y el resto, salvo los que reflejan muy poca). En función de la variable Ansiedad, las diferencias son significativas en la Media de Rendimiento Académico, en Lengua Castellana, en Matemáticas, en Ciencias Sociales, en Inglés y en Naturales. En este caso, el análisis de las pruebas post hoc indica una relación inversamente proporcional entre Ansiedad y Rendimiento (encontrándose entre los que manifiestan muy poca ansiedad y el resto). En el caso de la materia Religión las diferencias no fueron significativas.

Tabla io. ANova Rendimiento por Estados emocionales

\begin{tabular}{|c|c|c|c|c|c|c|c|}
\hline Variable & $\mathrm{N}$ & Perd & dos & Media & Des. Tip. & $\mathrm{F}$ & $\mathrm{P}$ \\
\hline \multicolumn{8}{|c|}{ Felicidad Total } \\
\hline \multirow{4}{*}{ Media Rendimiento } & 3 & o & Muy Poca Felicidad & 7.1667 & .23570 & \multirow{4}{*}{15.508} & \multirow{4}{*}{.000} \\
\hline & 46 & 28 & Poca Felicidad & 5.9215 & 2.02550 & & \\
\hline & 274 & IO8 & Bastante Felicidad & 6.4424 & I.79504 & & \\
\hline & $25 \mathrm{I}$ & 45 & Mucha Felicidad & 7.3787 & I. $674 \mathrm{I} 3$ & & \\
\hline \multicolumn{8}{|c|}{ Ansiedad Total } \\
\hline \multirow{3}{*}{ Media Rendimiento } & 305 & 78 & Muy Poca Ansiedad & 7.1119 & I. 70336 & \multirow{3}{*}{10.690} & \multirow{3}{*}{.000} \\
\hline & 237 & 83 & Poca Ansiedad & 6.3639 & I. 92194 & & \\
\hline & 35 & 20 & Bastante Ansiedad & $6.6 \mathrm{I} 42$ & I. 79807 & & \\
\hline
\end{tabular}

\section{Discusión-conclusiones}

A través de este apartado final se pretende dar respuesta a los objetivos que se marcaron al inicio de la investigación. De esta forma, se da respuesta al comportamiento de las variables de estudio, en función de las variables sociodemográficas consideradas (edad, género, cultura y estrato socioeconómico). Del mismo modo, se hace 
referencia a la existencia de relaciones entre las mismas, lo que se considerará en los siguientes párrafos.

En función del curso, se registran diferencias significativas en las puntuaciones totales y cada uno de los factores que integran la variable ansiedad. En este caso, se observa un descenso desde $3 .^{\circ}$ hasta $6 .^{\circ}$ de Primaria. De la misma manera, en felicidad se registran diferencias significativas para la edad, no solo para las puntuaciones totales, sino para cada una de las categorías que integran esta variable. En este caso, las diferencias se dan en sentido contrario al anteriormente comentado. Se puede afirmar que a medida que se va ascendiendo de curso, aumentan los niveles de felicidad. Se puede comenzar considerando la incidencia de los trastornos de ansiedad y su diagnóstico, observándose un descenso progresivo del diagnóstico de problemas de ansiedad con la edad del sujeto (Cazalla-Luna y Molero, 20I4 e Ingles et al., 20IO). Justo lo contrario acurre con la felicidad, siendo los primeros cursos los que evidencian un nivel más bajo. Esta realidad no entra dentro de lo esperado, dado que se podría intuir cierta independencia de ambas variables (felicidad y edad), ya que en Carballeira et al. (20I5) no se encontraron diferencias significativas en la felicidad en función de la variable que se está tratando.

Para la Ansiedad, en función del género, se vuelven a encontrar diferencias estadísticamente significativas para los totales, así como los factores Estados emocionales positivos, Sensaciones de Ansiedad y Estados emocionales negativos. Como ya se ha comentado, las personas pertenecientes al género masculino reflejan puntuaciones más elevadas en todos los casos en los que hay diferencias significativas. Esto demuestra que el género es otra de las variables sociodemográficas que se deben tener en cuenta en cualquier investigación que pretenda informar sobre los estados de ansiedad. El sentido de estas diferencias es contrario a los encontrados en varios trabajos consultados, dado que en ellos (Franco, Pérez y De Dios, 20I4; Moreno-Rosset et al., 2016; y Pulido y Herrera, 2016) son las mujeres (o niñas) las que evidencian niveles superiores en diferentes estados emocionales de carácter negativo. También aparecen trabajos en los que son los varones los que demuestran mayores niveles de ansiedad (Cazalla-Luna y Molero, 20I4). Continuando con la Felicidad, tanto en los totales como en las categorías Condiciones de vida positivas y Cambios en la vida, se vuelven a encontrar diferencias estadísticamente significativas. De nuevo, de forma contraria a lo que ocurre en el caso de la Ansiedad, son las mujeres las que demuestran niveles superiores en cuanto a la satisfacción personal (felicidad). Son varios los autores (Gómez, Villegas, Barrera y Cruz, 2007) que evidencian que las mujeres son más proclives a manifestar niveles superiores de felicidad, en comparación con los varones. En cualquier caso, el género es una variable influyente en estos estados emocionales. Como justificante de estas diferencias, se pretende un alejamiento de concepciones basadas en criterios de tipo biológico (Pinto, Dutra, Filgueiras, Juruena y Stingel, 2013). Sí se le concede mayor credibilidad a aquellas que tienen en cuenta diferencias en los patrones de socialización y la presencia marcada de determinados roles. Según esta consideración, el proceso de socialización llevado a cabo por familias, lo que pone de manifiesto la existencia de patrones de educación diferencial para ambos géneros. Esto justificaría que se pueda considerar el componente social como principal elemento responsable de las diferencias encontradas en esta variable (Caballero-Dominguez et al., 20I5; Pulido y Herrera, 20I6), así como aquellas concepciones centradas en considerar una mayor importancia de factores como el tipo de familia, el estado de salud, la edad y la 
religión sobre elementos ligados al género (Seligman, 20II). Todo ello es una forma de destacar la mayor relevancia del componente social y el proceso socializador.

Teniendo en cuenta las diferencias en ansiedad, en función de la cultura/religión y el estatus socioeconómico (Pulido y Herrera, 2016), aparecieron diferencias estadísticamente significativas en Ansiedad Total, Rasgos emocionales negativos, Estados emocionales positivos, Sensaciones de ansiedad y Estados emocionales negativos. Los pertenecientes a la cultura musulmana reflejan niveles superiores que los pertenecientes a la cristiana-occidental. Esta realidad también apareció en otros trabajos en los que se destaca este contexto cultural como elemento clave para el desarrollo emocional, lo que puede llegar a justificar estas diferencias entre ambos contextos, tal como se vio en múltiples estudios (Giménez-Dasí y Quintanilla, 2009; Gomez-Ortiz et al., 20I6; Rekart, Mineka, Zinbarg y Griffith, 2007; Pulido y Herrera, 2016). Para la variable Felicidad, se pueden describir diferencias estadísticamente significativas tanto en los niveles totales, como en los factores Condiciones de vida negativas, Satisfacción con la vida y Preocupaciones en la vida. El sentido de las mismas vuelve a ser inverso al que se describía para la ansiedad, siendo los musulmanes los que presentan niveles inferiores. En muchos de los trabajos consultados se refuerza la importancia concedida al contexto cultural y sus diferencias sobre el bienestar psicológico (Rodríguez, 20Io) como condicionantes de las respuestas emocionales positivas del sujeto (Jia et al., 2010; Paro, et al. 2010; Quiceno y Vinaccia, 2015). La religión y las normas aprendidas en el seno familiar tienen un importante efecto sobre los patrones ofrecidos dentro del seno familiar, en lo que a crianza de las personas se refiere (Soriano y González, 2013), lo que justifica estas diferencias en función de esta variable sociodemográfica.

Para concluir este apartado, se comentan las diferencias en ansiedad, en función del estatus socioeconómico. En este sentido, aparecieron diferencias estadísticamente significativas, en Ansiedad y los factores Estados emocionales positivos, Sensaciones de ansiedad, Estados emocionales negativos y Estados contrapuestos, observándose una distribución desorganizada en sus puntuaciones. En otros trabajos se ha encontrado un nivel superior de otros estados emocionales de carácter negativo entre los sujetos pertenecientes a estratos socioeconómicos más bajos (Pulido y Herrera, 20I6), algo que no concuerda con los resultados obtenidos. Por contra, para la variable Felicidad (las diferencias estadísticamente significativas se dan en Felicidad, Condiciones de vida negativas, Satisfacción con la vida y Condiciones de vida positivas), el sentido de las mismas vuelve a ser inverso. En trabajos como el de Quiceno y Vinaccia (2015) o el de Carballeira et al. (2015) se demuestra que alcanzan niveles superiores los sujetos pertenecientes al estrato socioeconómico alto. Esto se entiende considerando la concepción defendida por Alonso y Román (2014), según la cual el nivel económico y sociocultural correlaciona positivamente con el estilo educativo familiar, repercutiendo en diferentes dimensiones de desarrollo emocional, lo que vuelve a conducir a la importancia del contexto en el desarrollo emocional del sujeto.

Si se considera la variable Felicidad, en función de los niveles de ansiedad (Ansiedad Total) se pueden contemplar diferencias estadísticamente significativas, tanto para la Felicidad Total, como para cada uno de los factores. El comportamiento de ambas variables consideradas es contrario. Esta realidad apoya las diferencias encontradas, que indican una relación inversamente proporcional entre estados emocionales. Parece haber interferencia entre la presencia de estados emocionales positivos y los de carácter negativo (Carballeira et al., 20I5), llegándose a situar ambos estados 
como los polos opuestos. La ansiedad, en las etapas iniciales de la vida, puede dificultar el adecuado desarrollo del autoconcepto, suponiendo una falta de aceptación por parte de los demás (Delgado, Inglés y García-Fernández, 20I3) y dificultando el proceso de socialización (Piqueras, Espinosa-Fernández, García-López y Beidel, 20I2), lo que puede ir asociado a la evitación de muchas situaciones o la exposición a ellas sufriendo un intenso malestar (Gómez-Ortiz et al., 20I6), alejándose del funcionamiento óptimo del sujeto (Buceta-Fernández, Mas-García y Bueno-Palomino, 20I2). Por esta razón, los sujetos que experimentan menores niveles de ansiedad son los que reflejan niveles superiores en felicidad, habiendo interferencia entre ambos estados emocionales (Hjemdal, Vogel, Solem, Hagen y Stiles, 20II; Moreno-Rosset et al., 2016).

Tal como se pensaba, considerando la edad, no aparecieron diferencias significativas en cuanto al Rendimiento (solo se encontraron en materias específicas). Los resultados muestran una distribución desorganizada. El trabajo de Pulido y Herrera (2016) refleja diferencias en este sentido, coincidiendo con los resultados encontrados en este caso (distribución desorganizada). Considerando el género, se encontraron diferencias significativas, tanto desde el punto de vista de la Media, como en función de cada una de las materias. En todos los casos, las niñas evidencian un rendimiento superior. Para las diferencias entre géneros en cuanto al Rendimiento, fueron las mujeres las que presentan mejores resultados (Pulido y Herrera, 20I6). Con respecto a la cultura/religión, se comprueban diferencias estadísticamente significativas. En todos los casos, el grupo de alumnos pertenecientes a la cultura/ religión cristiana obtienen mejores resultados, realidad ya descrita en trabajos anteriores (Pulido y Herrera, 20I6). En este sentido, se mencionan las dificultades para la integración socio educativa (Oropesa, Moreno, Pérez y Muñoz-Tinoco, 20I4) y las diferencias en cuanto a la lengua materna entre musulmanes y cristianos, tal como informan algunos autores (Siqués y Vila, 20I4). Todos estos elementos se unen para provocar importantes problemas de integración socioeducativa del alumnado que no tiene el castellano como lengua madre. Considerando el Rendimiento en función del estatus, se encontraron diferencias significativas, tanto en la Media de Rendimiento como en cada una de las materias. En todos los casos, las calificaciones aumentan a medida que lo hace el estatus socioeconómico, realidad similar a la encontrada en Pulido y Herrera (2016).

Se pone fin a este apartado de discusiones relacionadas con la influencia de la ansiedad sobre el Rendimiento Académico. Tal y como se esperaba, se encontraron diferencias significativas en cuanto al Rendimiento, tanto en los totales como en todas las materias (Lengua Castellana, Matemáticas, Ciencias Sociales, Inglés y Naturales), con la excepción de Religión. En todos los casos, y después del análisis post hoc, se observan unas relaciones inversamente proporcionales. Por su parte, existe una clara influencia de la felicidad sobre el Rendimiento Académico. En todos los casos, los que reflejan niveles superiores de Felicidad presentan calificaciones más altas. Todo esto coincide con otros trabajos en los que sí se contempla una relación inversamente proporcional entre los niveles de ansiedad y el desarrollo educativo (Contreras et al., 2005). Por su parte, en el caso de la felicidad, la dirección de las diferencias encontradas en este trabajo coincide con las encontradas en otras experiencias (Salanova et al., 2005). Estos autores demostraron que los niveles más altos en rendimiento se relacionan con mayores niveles de autoeficacia, satisfacción y felicidad. Como 
justificante para estas diferencias, se considera la combinación de cansancio emocional, fatiga física, pérdida de interés por la actividad y baja realización personal (Schaufeli y Bakker, 2005).

De esta manera, el considerar y potenciar el desarrollo emocional posee grandes posibilidades en el ámbito educativo. Esta consideración permitiría señalado aumento de las fortalezas personales, aumentaría el bienestar y la felicidad, mejorando las competencias vitales (Barahona et al., 20I3). Esto puede llegar a ser un arma con la que afrontar la búsqueda de la mejora en el rendimiento académico, como ocurre con programas desarrollados en nuestro sistema educativo.

\section{Bibliografía}

Alonso, J. y Román, J. M. (20I4) Nivel sociocultural, prácticas educativas familiares y autoestima de los hijos en edades tempranas. Revista de Investigación Educativa, 32 (I), 187-202. Recuperado de http://dx.doi.org/Io.6oI8/rie.32.I.I 7342I.

Buceta-Fernández, J. M.; Mas-García, B. y Bueno-Palomino, A. (20I2) Intervención psicológica y salud: Control del estrés y conductas de riesgo. Madrid, España: Dykinson.

Barahona, M. N.; Sánchez, A. y Urchaga, J. D. (20I3) La Psicología Positiva aplicada a la educación: el programa cip para la mejora de las competencias vitales en la Educación Superior. Revista de Formación e Innovación Educativa Universitaria, 6 (4), 244-256. Recuperado de http://refiedu.webs.uvigo.es/Refied u/Vol64/REFIEDU645.pdf.

Caballero-Domínguez, C. C.; Suárez-Colorado, Y. P. y Brugesacarbono, H. D. (20I5) Characteristics of Emotional Intelligence in a Group of Higher Education Students with and without Suicidal Ideation. Revista CES Psicología, 8 (2), I38-155. Recuperado de https:// dialnet.unirioja.es/descarga/articulo/5236645.pdf.

Carballeira, M.; González, J. A. y Marrero, R. J. (20I5) Diferencias transculturales en bienestar subjetivo: México y España. Anales de Psicología, 3I (I), 199-206. http://dx.doi. org/ı.6or8/analesps.31.1.166931.

Cazalla-Luna, N. y Molero, D. (20I4) Inteligencia emocional percibida, ansiedad y afectos en estudiantes universitarios. Revista Española de Orientación y Psicopedagogía, 25 (3), 5673. Recuperado de http://dx.doi.org/I0.5944/reop.vol.25.num.3.20I4.13858.

Contreras, F.; Espinosa, J. C.; Esguerra, G.; Haikal, A. y Rodríguez, A. (2005) Autoeficacia, ansiedad y rendimiento académico en adolescentes. Diversitas: Perspectivas en psicología (I) 2, I83-I94 Recuperado de http://www.redalyc.org/articulo.oa?id=67910207.

Delgado, B.; Inglés, C. J. y García-Fernández, J. M. (20I3). Social anxiety and self-concept in adolescence. Revista de Psicodidáctica, I8, 179-194. Recuperado de http://www.ehu.eus/ ojs/index.php/psicodidactica/article/view/64II/62I4.

Franco, N.; Pérez, M. A. y De Dios, M. J. (20I4) Relación entre los estilos de crianza parental y el desarrollo de ansiedad y conductas disruptivas en niños de 3 a 6 años. Revista de Psicología Clínica con Niños y Adolescentes, I (2), I49-I56. Recuperado de http://www. revistapcna.com/sites/default/files/6-rpcna_vol.2.pdf.

Giménez-Dasí, M. y Quintanilla, L. (2009). «Competencia» social, «competencia» emocional: una propuesta para intervenir en Educación Infantil. Infancia y Aprendizaje, 32 (3), 359-373. Recuperado de http://dx.doi.org/I0.1174/0210370 09788964222.

Gómez, V.; Villegas, C.; Barrera, F. y Cruz, J. E. (2007) Factores predictores de Bienestar Subjetivo en una muestra colombiana. Revista Latinoamericana de Psicología, 39, 311-325. Recuperado de https://dialnet.unirioja.es/servlet/articulo?codigo $=2539736$.

Gómez-Castro, J. L. (1986) «Rendimiento escolar y valores interpersonales: Análisis de resultados en E.G.B. con el cuestionario siv de Leonardo V. Gordon». Bordón, 262, 257-275. 
Gómez-Ortiz, O.; Casas, C. y Ortega-Ruiz, R. (20i6) Ansiedad social en la adolescencia: factores psicoevolutivos y de contexto familiar. Behavioral Psychology/Psicología Conductual, 24 (I), 29-49.

González-Quiñones, J. C. y Restrepo, G. (20Io) Prevalencia de felicidad en ciclos vitales y relación con redes de apoyo en población colombiana. Revista de Salud Pública, I2, 228238. Recuperado de http://studylib.es/doc/5823123/ prevalencia-de-felicidad-en-ciclos-vitales-y-relaci\% $\mathrm{C}_{3} \% \mathrm{~B}_{3} \mathrm{n}$-con...

Gutiérrez, M. y Gonçalves, T. O. (20I3) Activos para el desarrollo, ajuste escolar y bienestar subjetivo de los adolescentes. International Journal of Psychology and Psychological Therapy, $\mathrm{I3}$ (3), 339-355.

Hjemdal, O.; Vogel, P. A.; Solem, S.; Hagen, K. y Stiles, T. C. (2oir) The relationship between resilience and levels of anxiety, depression, and obsessive-compulsive symptoms in adolescents. Clinical Psychology \& Psychotherapy, I8, 314-321. Recuperado de https://doi. org/IO.IOO2/cpp.719.

JiA, Z.; Tian, W.; He, X.; LiU, W.; Jin, C. y Ding, H. (20I0) Mental health and quality of life survey among child survivors of the 2008 Sichuan earthquake. Quality of Life Research, 19 , I38I-I39I. Recuperado de https://doi.org/Io.I007/sıin36-0IO-9703-8.

Moreno-Rosset, C.; Arnal-Remón, B.; Antequera-Jurado, R. y Ramírez-Uclés, I. (2016) Anxiety and psychological wellbeing in couples in transition to parenthood. Clínica y Salud, 27, 29-35. Recuperado de http://dx.doi.org/Io.Ior6/j.clysa. 2016.0I.004.

Oropesa, F.; Moreno, C.; Pérez, P. y Muñoz-Tinoco, V. (20I4) Rutinas de tiempo libre: oportunidad y riesgo en la adolescencia. Cultura y Educación, 26 (I), I59-183. Recuperado de http://dx.doi.org/IO.I080/II356405.2014.908670.

Ortuño-Sierra, J.; García-Velasco, L.; Inchausti, F.; Debbané, M. y Fonseca-Pedrero, E. (2016) Nuevas aproximaciones en el estudio de las propiedades psicométricas del STAI. Actas Españolas de Psiquiatría, 44 (3), 83-92. Recuperado de https://www.actaspsiquiatria. es/repositorio/18/ıor/ESP/18-Ior-ESP-83-92-48820I.pdf.

Perandones, T. M. (2015) Influencia de variables personales en la docencia. Relación de las percepciones de autoeficacia del profesorado con sus fortalezas y virtudes, felicidad, sentido del humor y personalidad. Granada: Universidad de Granada.

Paro, H.; Morales, N.; Silva, C.; Rezende, C.; Pinto, R.; Morales, R.; et al. (2OIo) Healthrelated quality of life of medical students. Medical Education, 44, 227-235. Recuperado de http://dx.doi.org/Io.IIII/j.1365-2923.2009.03587.x.

Pinto, B. M. D. C.; Dutra, N. B.; Filgueiras, A.; Juruena, M. F. P. y Stingel, A. M. (2013) Diferenças de gênero entre universitários no reconhecimento de expressões faciais emocionais. Avances en Psicología Latinoamericana, 3I (I), 200-222. Recuperado de https://revistas. urosario.edu.co/index.php/apl/article/view/2402/2094.

Piqueras, J. A.; Espinosa-Fernández, L.; García-López, L. J. y Beidel, D. C. (20i2) Validación del «Inventario de ansiedad y fobia social forma breve» (SPAI-в) en jóvenes adultos españoles. Behavioral Psychology/Psicología Conductual, 20, 505-528.

Pulido, F. y Herrera, F. (20I6) El miedo como predictor del rendimiento académico: El contexto pluricultural de Ceuta. Revista Española de Orientación y Psicopedagogía, 27 (2), 29-44. Recuperado de http://www2.uned.es/reop/pdfs/2016/27-2\%20-\%2oPulido.pdf.

Quiceno, J. M. y Vinaccia, S. (2015) Calidad de vida, fortalezas personales, depresión y estrés en adolescentes según sexo y estrato. International Journal of Psychology and Psychological Therapy, I4 (2), I55-170. Recuperado de http://studylib.es/doc/4485123/calidad-de-vi$\mathrm{da}$--fortalezas-personales--depresi $\% \mathrm{C}_{3} \% \mathrm{~B}_{3} \mathrm{n}-\mathrm{y}$-estr\% $\mathrm{C}_{3} \% \mathrm{~A}_{9} \ldots$

Rekart, K. N.; Mineka, S.; Zinbarg, R. E. y Griffith, J. W. (2007) Perceived family environment and symptoms of emotional disorders: The role of perceived control, attributional style, and attachment. Cognitive Therapy and Research, 3I (4), 4I9-436. http://dx.doi. org/I0.1007/sIo608-007-9I3I-4. 
Rodríguez, C. (20I0) Variables psicológicas asociadas con la felicidad en centros peri-urbanos y urbanos marginales de Lima. UCV-Scientia, 2 (I), 6I-68. Recuperado de http://docplayer.es/15156556-Variables-psicologicas-asociadas-con-la-felicidad-en-centros-peri-urbanos-y-urbanos-marginales-de-lima.html.

Salanova, M.; Martínez, I. M.; Bresó, E.; Llorens, S. y Grau, R. (2005) Bienestar psicológico en estudiantes universitarios: facilitadores y obstaculizadores del desempeño académico. Anales de psicología, 2I (I), I70-180. Recuperado de http://www.um.es/analesps/v2I/ V2I_I/I6-2I_I.pdf.

Schaufeli, W. B. y Bakker, A. B. (2004) Job demands, job resources and their relationship with burnout and engagement: A multi-sample study. Journal of Organizational Behavior, 25, 293-315. Recuperado de http://dx.doi.org/IO.IOO2/ job.248.

Seligman, M. (20II) La auténtica felicidad. España: Byblos.

SiquÉs, C. y VILA, I. (20I4) Estrategias discursivas y aprendizaje de la lengua escolar: un estudio etnográfico en un aula de alta diversidad étnica y lingüística. Cultura y Educación, 26 (2), 349-376. Recuperado de http://dx.doi.org/IO.I080/II356 405.20I4.935II2.

Soriano, E. y GONZÁlez, A. J. (2013) Las competencias emocionales en el ámbito afectivo-sexual de los adolescentes autóctonos e inmigrantes. Revista de Investigación Educativa, 31 (I), I33-I49. http://dx.doi.org/ro.6or8/rie.31.1.I50 53I.

Spielberger, C. D. (200I) STAic. Cuestionario de Ansiedad Estado/Rasgo en niños. Madrid: TEA. 
